Supplement of

\title{
A Vacuum Ultraviolet Ion Source (VUV-IS) for Iodide-Chemical Ionization Mass Spectrometry: A Substitute for Radioactive Ion Sources
}

5 Yi Ji et al.

Correspondence to: L. Gregory Huey (greg.huey@eas.gatech.edu) 


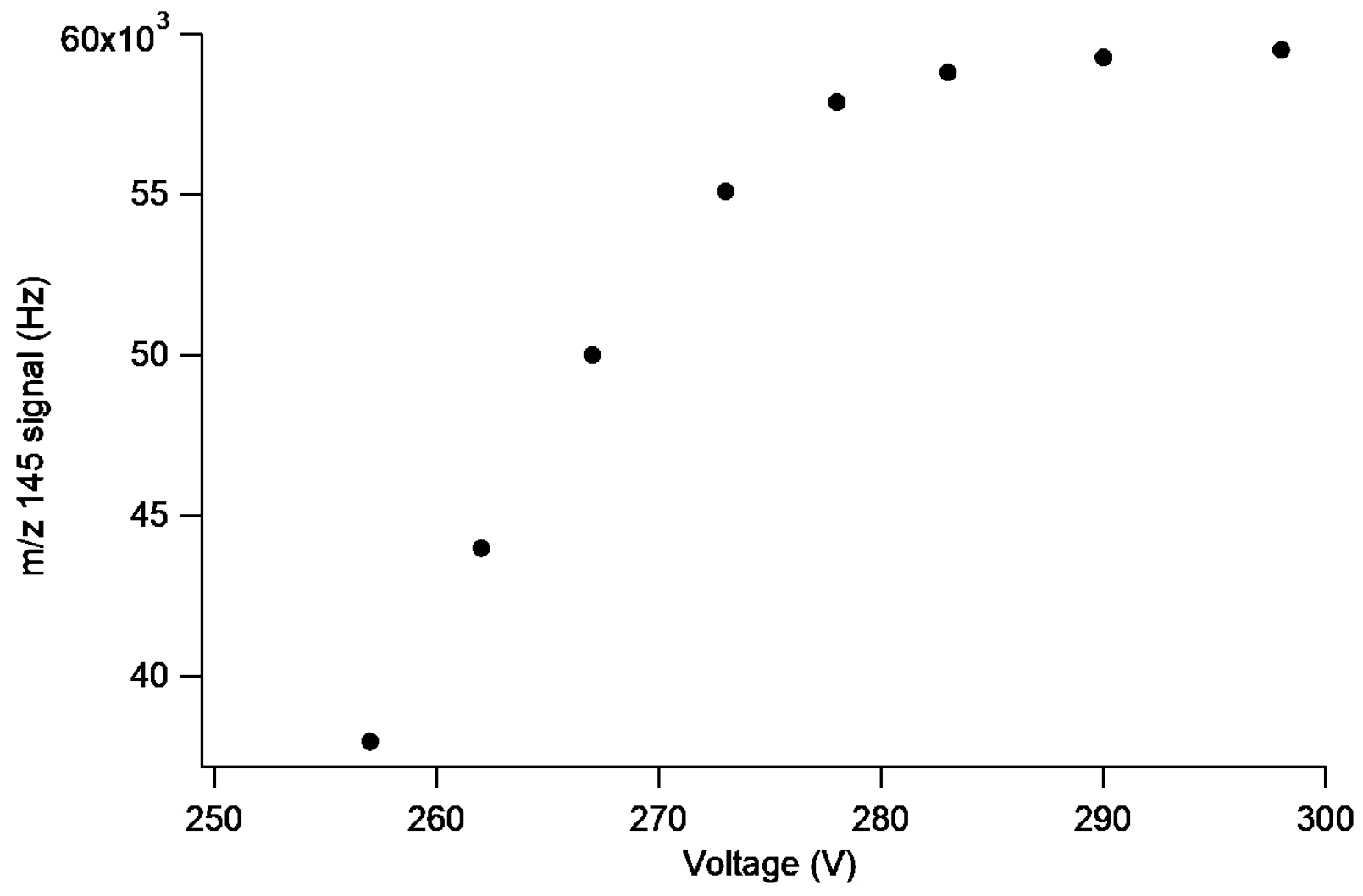

Figure S1. CIMS ion current at $\mathrm{m} / \mathrm{z} 145\left(\mathrm{I}^{-}\left(\mathrm{H}_{2} \mathrm{O}\right)\right)$ as a function of voltage across the krypton lamp. Note that the lamp ignites at voltage of $\sim 280 \mathrm{~V}$. 
(a)

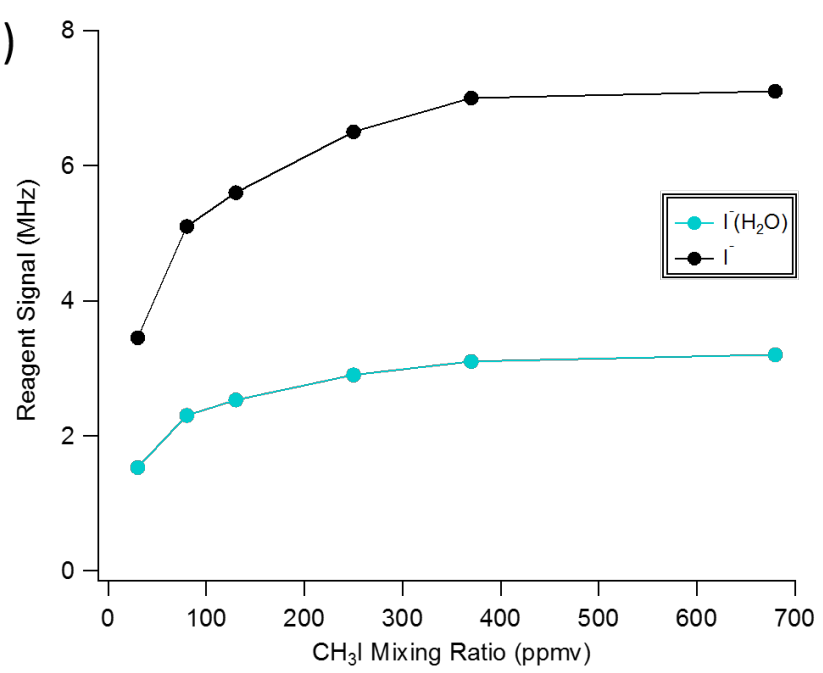

0
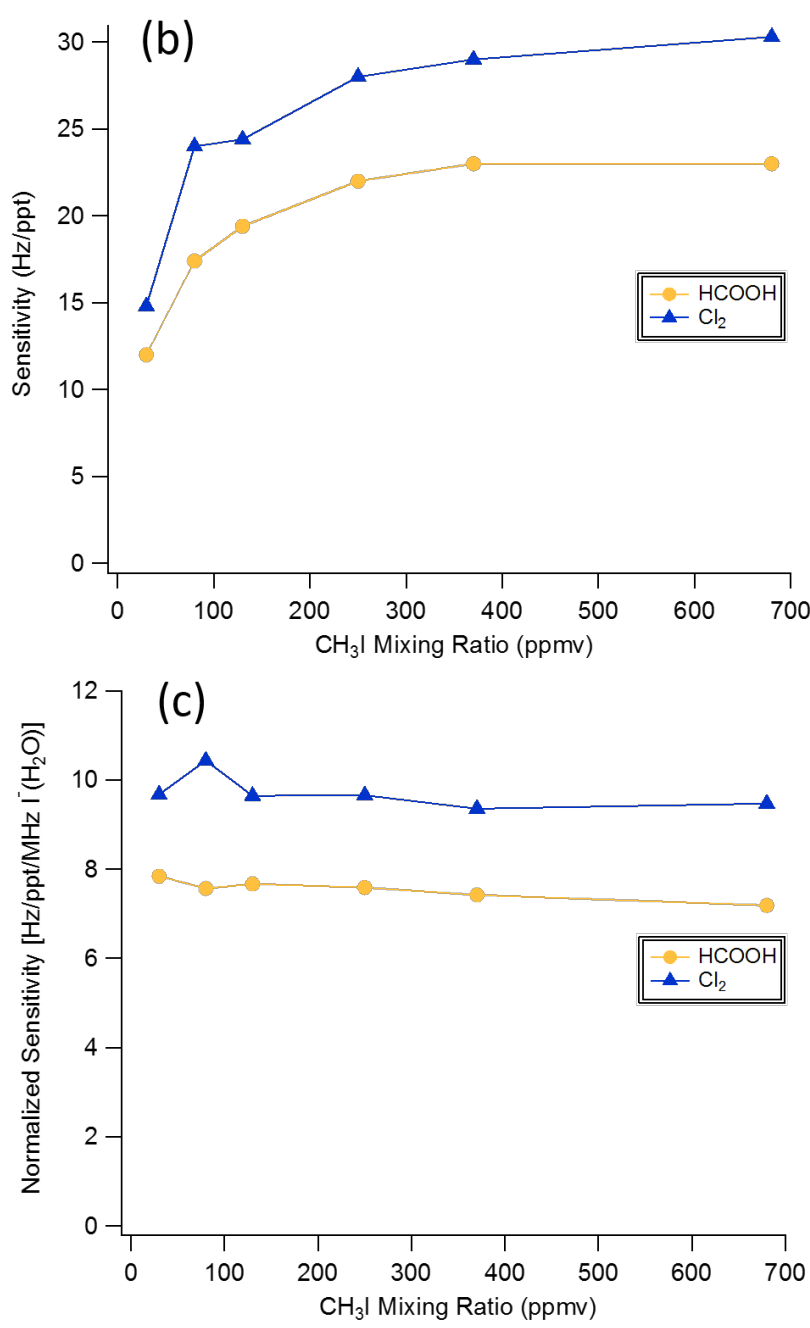

Figure S2. TOF-CIMS (a) reagent signal levels (b) sensitivity (c) normalized sensitivity as a function of $\mathrm{CH}_{3} \mathrm{I}$ at 30 torr. 


\section{Sample Calculation of Absorption of VUV light by $\mathrm{CH}_{3} \mathrm{I}$}

To calculate how much of the VUV light is absorbed, the Beer-Lambert Law is applied,

$$
\frac{I(\lambda)}{I_{0}(\lambda)}=\exp (-\sigma(\lambda) n L)
$$

where $I(\lambda)$ is the intensity of light at wavelength $\lambda$ after absorption, $I_{0}(\lambda)$ is the original light intensity at

30 wavelength $\lambda, \sigma(\lambda)$ is the absorption cross section of the absorber molecule at wavelength $\lambda, n$ is number concentration of the absorber molecule, and $L$ is the path length over which the light can be absorbed.

Sample calculation:

For $86.5 \mathrm{ppmv}$ of $\mathrm{CH}_{3} \mathrm{I}$ at a pressure of 20 torr

35

$$
n=5.70 \times 10^{13} \text { molecule } \mathrm{cm}^{-3}
$$$$
\sigma(\lambda)=7 \times 10^{17} \mathrm{~cm}^{2} \text { molecule }{ }^{-1}
$$

$L=21 \mathrm{~cm}$

$$
\frac{I(\lambda)}{I_{0}(\lambda)}=\exp (-\sigma(\lambda) n L)=0.92
$$

Therefore, in this example $\sim 8 \%$ of the light emitted from the VUV lamp is absorbed by the methyl 40 iodide. 